\title{
Evaluating adherence to recommended diets in adults: the Alternate Healthy Eating Index
}

\author{
Marjorie L McCullough ${ }^{1, *}$ and Walter C Willett ${ }^{2}$ \\ 'Epidemiology and Surveillance Research, American Cancer Society, 1599 Clifton Road NE, Atlanta, \\ GA 30329-4251, USA: ${ }^{2}$ Harvard School of Public Health, Departments of Nutrition, Epidemiology, and the \\ Channing Laboratory, Department of Medicine, Brigham and Women's Hospital and Harvard Medical School, \\ Boston, MA 02115, USA
}

\begin{abstract}
Objective: The Healthy Eating Index (HEI), designed to assess adherence to the Dietary Guidelines for Americans and the Food Guide Pyramid, was previously associated with only a small reduction in major chronic disease risk in US adult men and women. We assessed whether an alternate index would better predict risk.

Design: Dietary intake reported by men and women from two prospective cohorts was scored according to an a priori designed Alternate Healthy Eating Index (AHEI). In contrast with the original HEI, the AHEI distinguished quality within food groups and acknowledged health benefits of unsaturated oils. The score was then used to predict development of CVD, cancer or other causes of death in the same population previously tested.

Subjects: 67271 women from the Nurses' Health Study and 38615 men from the Health Professionals' Follow-up Study.

Results: Men and women with AHEI scores in the top vs. bottom quintile had a significant $20 \%$ and $11 \%$ reduction in overall major chronic disease, respectively. Reductions were stronger for CVD risk in men $(\mathrm{RR}=0.61,95 \% \mathrm{CI} 0.49-0.75)$ and women ( $\mathrm{RR}=0.72,95 \% \mathrm{CI} 0.60-0.86$ ). The score did not predict cancer risk.

Conclusions: The AHEI was twice as strong at predicting major chronic disease and CVD risk compared to the original HEI, suggesting that major chronic disease risk can be further reduced with more comprehensive and detailed dietary guidance.
\end{abstract}

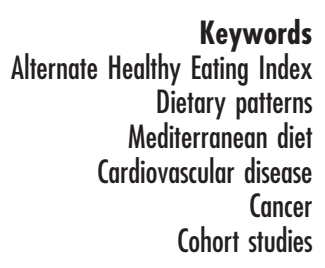

Cardiovascular disease (CVD) and cancer are the two leading causes of death in the USA ${ }^{1}$. Lifestyle factors, including diet, are thought to play an important role in their prevention ${ }^{2}$. An overall diet that includes several purported healthy factors, and lacks unhealthy factors, is likely to have more of an impact on disease outcome than any one dietary factor alone ${ }^{3}$. Investigators have thus begun using dietary scores to characterise a diet that simultaneously reflects multiple healthful behaviours ${ }^{4,5}$. Greater adherence to healthy diet patterns, as measured using diet scores, has been associated with lower mortality in different populations $^{6-8}$, including the Mediterranean?.

In the USA, the Dietary Guidelines for Americans (DGA) represent the primary dietary advice given to Americans on avoiding major chronic disease ${ }^{10}$. These guidelines are updated every five years ${ }^{11}$. In order to track adherence to the 1995 DGA and the Food Guide Pyramid ${ }^{12}$ (its visual counterpart), the United States Department of Agriculture developed the Healthy Eating Index $(\mathrm{HEI})^{13}$. This 10-component, 100-point score measures how well the diets of all Americans conform to recommendations for consumption of foods from five food groups, as well as guidelines on fat, cholesterol, and sodium, and dietary variety.

We previously tested whether having higher HEI scores predicted lower chronic disease risk in two large cohorts of men and women in the USA The score weakly predicted major chronic disease risk in men, but not in women ${ }^{14,15}$. Men whose diets fell into the highest HEI quintile (vs. lowest) were at $11 \%$ lower risk of overall chronic disease (relative risk $(\mathrm{RR})=0.89,95 \%$ confidence interval $(\mathrm{CI})$ 0.79-1.00) but women were not at lower risk $(\mathrm{RR}=0.97,95 \%$ CI 0.891.06). A statistically significant lower risk of CVD was observed in men with the highest HEI scores ( $R R=0.72$, 95\% CI 0.60-0.88) but the association was weaker in women $(\mathrm{RR}=0.86,95 \% \mathrm{CI} 0.72-1.03)$. The score did not predict cancer risk in men or women. The associations with major chronic disease may have been improved had there been distinction between unsaturated and saturated fats, the form of carbohydrate, or protein sources (e.g. processed meats vs. fish), in addition to other components. Animal and human data suggest that greater risk reduction could be achieved with such changes ${ }^{16-21}$. 
We therefore developed an Alternate Healthy Eating Index (AHEI) to address these concerns. In this paper, we describe our previous work on development and testing of the $\mathrm{AHEI}^{22}$, and compare our score with the Mediterranean diet index.

\section{Methods}

\section{Study populations}

As described in detail elsewhere ${ }^{22}$, we analysed data collected from two large ongoing cohorts of men and women. In 1986, 51529 men aged 40-75 years were enrolled in the Health Professionals' Follow-up Study (HPFS), a prospective investigation of dietary aetiologies of heart disease and cancer. The Nurses' Health Study (NHS) began in 1976 and included 121700 female nurses aged 30-55 years. In 1984, 81757 of these women completed an extensive food-frequency questionnaire (FFQ) (similar to HPFS). We excluded men and women with previously diagnosed heart disease, cancer or chronic renal failure and those who did not complete an FFQ or who reported implausible energy intakes. The final analytic cohorts included 38615 men and 67271 women. At baseline, participants provided anthropometric, lifestyle and medical information. Every two years we sent follow-up questionnaires to obtain up-to-date information on risk factors and to identify newly diagnosed diseases; most dietary information was updated every four years.

\section{Dietary assessment}

Dietary intake data were collected in 1986 and 1990 in men, and in 1984, 1986 and 1990 in women using a validated, semi-quantitative FFQ which contains approximately 130 questions (varied slightly from year to year) ${ }^{23-28}$. A common serving (svg) size of food or beverage was specified on the FFQ (e.g. $1 / 2$ cup carrots or 2 slices bacon) and participants were asked how often, on average, they consumed this amount over the previous year. Nine possible frequency responses ranged from 'never or less than once per month' to 'six or more times per day'. Information on types of fats and oils used in cooking, brand of cold cereal typically consumed, and brand and frequency of multivitamin supplements was also collected. We calculated nutrient intakes by multiplying the consumption frequency of each food by the nutrient content of specified portions, and then summing nutrient contributions from all foods. Nutrient values were obtained from the Harvard University Food Composition Database.

\section{The Alternate Healthy Eating Index}

AHEI variables and scoring decisions were made $a$ priori, by discussion with nutrition researchers, to capture specific dietary patterns and eating behaviours consistently associated with lower chronic disease risk in clinical and epidemiologic investigations. The AHEI includes nine components, including some components from the original $\mathrm{HEI}^{13}$, such as fruits and vegetables (however, we removed potatoes and potato products from the vegetable component). Eight of the nine components contributed $0-10$ points to the total score (10 indicated that recommendations were met, zero that they were not). Intermediate intakes were scored proportionally between 0 and 10 . Criteria for scoring the AHEI were as follows: higher scores were given for a greater intake of vegetables (10 points for $5+$ svgs/day; 0 points for no svgs/day) and fruit (10 points for $4+$ svgs/ day; 0 points for no svgs/day). The AHEI also provides more detail for scoring diet quality of several other food groups. For example, whereas the original HEI gave more credit for consumption of any type of meat, we assigned higher scores for consuming more fish and poultry vs. red or processed meat. The ratio of white to red meat was intended to capture a replacement of white for red meat (10 points for 4:1 ratio; 0 points for ' 0 ' - except vegetarians received a score of 10). We also included a separate component for non-meat protein sources, including nuts and soy products (10 points for $1+$ svgs/day; 0 points for no svgs/day). To capture a higher intake of whole grains, we gave credit for higher cereal fibre intake (10 points for $15+\mathrm{g}$ /day; 0 points for no $\mathrm{g}$ /day). The ratio of polyunsaturated to saturated fat was calculated to capture higher consumption of beneficial unsaturated oils common in the USA (10 points for a ratio $\geq 1$; none for $<0.1$ ). A low trans fat intake also received a higher score (10 points for $\leq 0.5 \%$ $\mathrm{kcal}$; 0 points for $\geq 4 \% \mathrm{kcal})$. Moderate alcohol consumption contributed to higher points (10 points for $1.5-2.5 \mathrm{svgs} /$ day men and $0.5-1.5 \mathrm{svgs} /$ day women; 0 points for either no consumption or $>3.5 \mathrm{svgs} /$ day men and $>2.5 \mathrm{svgs}$ (day women). A long-term multivitamin component was dichotomous, contributing either 7.5 points (for regular use $>5$ years) and 2.5 points (for all others) to avoid over-weighting this component. All individual component scores were summed for a total AHEI score ranging from 2.5 (worst) to 87.5 (best). More detail on the rationale for the components used is provided in the original manuscript ${ }^{22}$.

\section{Outcome ascertainment}

The primary endpoint for this study, 'major chronic disease', was defined as the initial occurrence of CVD or cancer or non-trauma-related death. We also examined the associations of the scores with CVD and cancer risk separately. We were particularly interested in whether the AHEI was related to disease incidence, because this is more relevant for disease prevention than using mortality as an outcome.

CVD was defined as fatal or non-fatal myocardial infarction (MI), fatal or non-fatal stroke, or sudden death. 
We asked all men and women who reported incident MI or stroke on their biennial questionnaires to confirm the report and to provide permission for the review of medical records. Self-reports were confirmed using established criteria $^{29,30}$. We included all confirmed cancers except non-melanoma skin cancer, in situ breast cancer and lowgrade, organ-confined prostate cancer (stage A or B, and Gleason grade $<7$ ), because of the relatively low mortality from these highly prevalent lesions.

We included deaths, except those from external causes (e.g. injuries and suicides), in the composite major chronic disease endpoint. Deaths were reported by next of kin, co-workers, or postal authorities, or ascertained by a search for non-respondents using the National Death Index ${ }^{31}$. Non-responding participants were assumed to be alive if not listed in the National Death Index.

\section{Statistical analyses}

Each participant contributed follow-up time from the return of his or her baseline questionnaire until the date of CVD, cancer, or death, or until 1 February 1994 (men) or 1 June 1996 (women). Overall follow-up, on the basis of eligible person-years, was $>95 \%$ complete for both men and women.

Quintiles of the AHEI were defined using a cumulative average scoring method, to maximally utilise the repeated diet assessments ${ }^{32}$. For example, in men, the 1986 AHEI score was used to predict outcome in 1986-1990, and an average of the 1986 and 1990 AHEI scores were related to outcome between 1990-1994. If no questionnaire was completed in 1990, the 1986 AHEI score was carried forward. We did not update diet for participants who had a new diagnosis of angina, hypercholesterolaemia, diabetes, or hypertension because potential changes in diet as a result of these diagnoses may confound the association between diet and disease.

We calculated relative risks (RR) as the incidence rate of major chronic disease among participants in each quintile of the diet quality scores divided by the incident rate for those in the lowest quintile, adjusting for age. To adjust simultaneously for several risk factors, we used pooled logistic regression ${ }^{33}$, which accounts for changes in covariates over time and has been shown to be a close approximation to Cox proportional hazard analysis ${ }^{34}$. A trend test was computed using the median values for quintiles modelled as a single continuous variable.

Covariates included the following major determinants of health: age, cigarette smoking, body mass index $\left(\mathrm{kg} / \mathrm{m}^{2}\right)$, leisure-time physical activity (in metabolic equivalents, or METs), total energy intake and in women, postmenopausal hormone use. In addition, hypercholesterolaemia and hypertension were included as covariates in the CVD and major chronic disease models and vitamin $\mathrm{E}$ was included only in the CVD models. All reported $P$-values are two-sided.

\section{Results}

During the period 1986-1994, we documented 3119 major chronic disease endpoints in men, which included 1092 CVD events, 1661 cancers, and 366 deaths not resulting from CVD or cancer. In women, 7077 chronic disease outcomes occurred from 1984-1996; these included 1365 CVD events, 5216 cancers, and 496 deaths not resulting from CVD or cancer.

Men and women with higher AHEI scores were less likely to smoke, exercised more and were slightly older. They also reported higher energy intakes, likely due in part to increased physical activity (not shown).

The association between the AHEI and risk of major chronic disease, CVD, and cancer in men is provided in Table 1. After controlling for smoking and other known risk factors, we observed a moderate inverse relation with overall major chronic disease $(\mathrm{RR}=0.80,95 \% \mathrm{CI}, 0.71-$ $0.91, P<0.001)$. Adjusting for other risk factors, men with highest AHEI scores had a 39\% lower CVD risk than those with lowest scores $(\mathrm{RR}=0.61,95 \%$ CI $0.49-0.75)$; however, the AHEI did not predict cancer risk. Results were similar when body mass index was not included in the model. The overall findings for women were weaker than for men, but the AHEI predicted a significant reduction in major chronic disease risk in our multivariate models ( $\mathrm{RR}=0.89,95 \% \mathrm{CI} 0.82-0.96, P=0.009)$. Highest (compared to lowest) AHEI scores were related to a $28 \%$ lower CVD risk in women $(\mathrm{RR}=0.72,95 \%$ CI $0.60-0.86$, $P<0.001$ ). Again, we observed no association between AHEI and cancer risk in women.

Because alcohol consumption is known to cause injuryrelated death, and many people do not consume alcohol, we conducted additional analyses leaving traumatic deaths in the major chronic disease outcome, and also evaluated the score excluding the alcohol component. These changes had no material influence on the association with major chronic disease (data not shown).

\section{Discussion}

In these two cohorts of men and women, individuals whose diets were most consistent with the AHEI goals had a $20 \%$ and $11 \%$ lower risk of overall major chronic disease, respectively. CVD risk was statistically significantly lower in both men (39\%) and women (28\%) for individuals with scores in the highest compared to the lowest AHEI quintile. These associations were approximately double those previously observed using the original HEI in the same population. However, neither the original HEI nor the AHEI predicted overall incident cancer risk. The implications of this study are that dietary scores can be used to assess health outcomes associated with adherence to dietary recommendations, and diet scores, and the guidelines on which they are based, can be improved to strengthen chronic disease risk reduction. 
Table 1 Relative risk (RR) and 95\% confidence intervals of major chronic disease, cardiovascular disease and cancer in men and women according to the Alternate Healthy Eating Index (AHEI)

\begin{tabular}{|c|c|c|c|c|c|c|}
\hline & \multicolumn{6}{|c|}{ Quintiles of AHEI scores } \\
\hline & 1 & 2 & 3 & 4 & 5 & $P$ trend $^{*}$ \\
\hline \multicolumn{7}{|l|}{ Major chronic disease $†$} \\
\hline Men: RR $\ddagger$ & 1.0 & $\begin{array}{l}0.96 \\
(0.86-1.07)\end{array}$ & $\begin{array}{l}0.88 \\
(0.79-0.99)\end{array}$ & $\begin{array}{l}0.79 \\
(0.71-0.89)\end{array}$ & $\begin{array}{l}0.8 \\
(0.71-0.91)\end{array}$ & $<0.001$ \\
\hline Women: RR $\ddagger$ & 1.0 & $\begin{array}{l}0.97 \\
(0.90-1.04)\end{array}$ & $\begin{array}{l}0.92 \\
(0.88-0.99)\end{array}$ & $\begin{array}{l}0.95 \\
(0.87-1.02)\end{array}$ & $\begin{array}{l}0.89 \\
(0.82-0.96)\end{array}$ & 0.009 \\
\hline \multicolumn{7}{|l|}{ Cardiovascular disease§ } \\
\hline Men: RR $\ddagger$ & 1.0 & $\begin{array}{l}0.85 \\
(0.71-1.00)\end{array}$ & $\begin{array}{l}0.79 \\
(0.66-0.95)\end{array}$ & $\begin{array}{l}0.67 \\
(0.56-0.81)\end{array}$ & $\begin{array}{l}0.61 \\
(0.49-0.75)\end{array}$ & $<0.001$ \\
\hline Women: RR $\ddagger$ & 1.0 & $\begin{array}{l}0.95 \\
(0.82-1.11)\end{array}$ & $\begin{array}{l}0.80 \\
(0.68-0.94)\end{array}$ & $\begin{array}{l}0.75 \\
(0.63-0.89)\end{array}$ & $\begin{array}{l}0.72 \\
(0.60-0.86)\end{array}$ & $<0.001$ \\
\hline \multicolumn{7}{|l|}{ Cancerף } \\
\hline Men: RR $\ddagger$ & 1.0 & $\begin{array}{l}1.10 \\
(0.94-1.28)\end{array}$ & $\begin{array}{l}0.99 \\
(0.85-1.16)\end{array}$ & $\begin{array}{l}0.94 \\
(0.80-1.10)\end{array}$ & $\begin{array}{l}1.03 \\
(0.87-1.22)\end{array}$ & 0.66 \\
\hline Women: RR $\ddagger$ & 1.0 & $\begin{array}{l}0.94 \\
(0.86-1.03)\end{array}$ & $\begin{array}{l}1.03 \\
(0.95-1.13)\end{array}$ & $\begin{array}{l}1.04 \\
(0.95-1.13)\end{array}$ & $\begin{array}{l}1.00 \\
(0.92-1.11)\end{array}$ & 0.39 \\
\hline
\end{tabular}

${ }^{*} P$-value, test for trend over quintiles of index scores using the median value per quintile.

$\dagger$ Major chronic disease $=$ CVD, cancer, or death, whichever came first.

$\ddagger$ Adjusted for age (5-year categories), smoking (never, past, 1-14 cigarettes per day, 15 to 24 cigarettes per day, $>25$ cigarettes per day), time period, body mass index (quintiles), physical activity (six categories of METs), total energy intake (quintiles), postmenopausal hormone use (women), and, in all except cancer models, history of hypertension or hypercholesterolaemia at baseline. The CVD model includes vitamin E.

$\S$ Cardiovascular disease $=$ fatal or non-fatal myocardial infarction, stroke, or sudden death .

I Cancer = all cancers except non-melanoma skin cancers, in situ breast cancers and non-aggressive prostate cancers.

The major differences between the AHEI and the original HEI included specific attention to fat quality and food group quality (e.g. meat sources, whole grains). The HEI gave high scores for total fat reduction $(<30 \%$ of calories) and did not recognise the benefits of consuming unsaturated oils, despite strong evidence that unsaturated fats have beneficial effects on heart disease and lipid profiles ${ }^{16,17,19,35}$. We therefore wanted to give appropriate credit for a healthful dietary fatty acid composition in the AHEI. We did not include monounsaturated fats because they are more commonly consumed as olive oil in the Mediterranean than the USA ${ }^{36}$, where the major sources are red meat and dairy fat. Polyunsaturated oils common in the US diet were therefore targeted. The original HEI also did not distinguish carbohydrate quality or quality of protein sources. We added cereal fibre and a 'white' (poultry and fish) vs. 'red' meat ratio to address quality within these food groups.

Table 2 provides a comparison of the original HEI, the AHEI and the Mediterranean index. The components included in these scores vary slightly from one another, reflecting different food consumption patterns and interpretation of science by the developers of each index. Some components are similar across all scores (e.g. fruits and vegetables), while other components are completely different. For example, dairy is considered beneficial in the HEI score, moderation is considered beneficial in the Mediterranean index (where most dairy is

Table 2 Qualitative comparison of the Healthy Eating Index (HEI), the Alternate Healthy Eating Index (AHEI) and the Mediterranean Diet index*

\begin{tabular}{|c|c|c|c|}
\hline Component & HEl† & Alternate HEI & Mediterranean§ \\
\hline Dairy products & $\uparrow$ & - & $\downarrow$ \\
\hline Vegetables & $\uparrow$ & $\uparrow$ (no potatoes) & $\uparrow$ \\
\hline Fruit & $\uparrow$ & $\uparrow$ & $\uparrow$ \\
\hline Nuts, seeds & $\uparrow(w /$ meat $)$ & $\uparrow$ & $\uparrow(w /$ fruit $)$ \\
\hline Bread/grains & $\uparrow$ & $\uparrow$ cereal fibre & $\uparrow$ \\
\hline Meat, poultry \& fish & $\uparrow$ & $\uparrow$ fish/poultry to red meat ratio & $\downarrow$ meat \& poultry; $\uparrow$ fish \\
\hline Cholesterol & $\downarrow$ & - & - \\
\hline Fat & $\downarrow$ tot \& SF & $\begin{array}{l}\uparrow \mathrm{P}: \mathrm{S} \text { ratio } \\
\downarrow \text { trans fat }\end{array}$ & $\uparrow \mathrm{M}: \mathrm{S}$ ratio \\
\hline Sodium & $\downarrow$ & - & - \\
\hline Alcohol & - & $\uparrow$ moderate & $\uparrow$ \\
\hline Multivitamins & - & $\uparrow$ & - \\
\hline
\end{tabular}

*Arrows indicate general direction of recommended intake; parentheses provide additional details on scoring method, for example where the component is included in the overall diet score.

† Kennedy et al. ${ }^{13}$.

$\ddagger$ McCullough et al. ${ }^{22}$

$\S$ Trichopolou et al. ${ }^{9}$. 
consumed in high-fat form), and is not included in the AHEI. The latter two indices share in common attention to beneficial oil consumption, and both specifically credit higher fish, nuts, and moderate alcohol consumption.

There are several possible reasons why we observed no association with cancer with either the HEI or the AHEI. In general, more is known about diet and CVD than about diet and cancer and each component had a hypothetical association with CVD, while only about half are related to cancer risk. If specific types of fruits and vegetables most strongly predict cancer, their effects would be diluted by pooling all fruits and vegetables together.

Newer hypotheses for cancer, including lycopene ${ }^{37}$, calcium $^{38}$ and vitamin $\mathrm{D}^{39}$, were not included in the score because they were less established at the time of score development. Cancer is a constellation of several diseases, whereas CVD is a more 'homogeneous' endpoint. The temporal relationships between diet and cancer are also much less clear than for CVD. Further research should continue to examine dietary factors and patterns for cancer prevention.

In summary, the Alternate Healthy Index was shown to be twice as strong as the original HEI in predicting overall chronic disease risk in US men and women, primarily driven by a marked inverse relation with cardiovascular disease. Differences between the original and alternate HEI included attention to fat quality, with an emphasis on choosing unsaturated over saturated fats, as well as inclusion of alcohol, cereal fibre, and fish in the diet. These qualities overlap in many ways with the healthy diet consumed in Mediterranean regions.

\section{References}

1 National Center for Health Statistics. National Vital Statistics Reports. Atlanta, GA: Centers for Diseases Control and Prevention, 2003.

2 Willett WC. Diet and health: What should we eat? Science 1994; 264: 532-7.

3 Sacks FM, Obarzanek E, Windhauser MM, Svetky LP, Vollmer WM, McCullough MM, et al. Rationale and design of the Dietary Approaches to Stop Hypertension trial (DASH). A multicenter controlled-feeding study of dietary patterns to lower blood pressure. Annals of Epidemiology 1995; 5: $108-18$.

4 Kant AK. Indexes of overall diet quality: a review. Journal of the American Dietetic Association 1996; 96: 785-91.

5 Hu FB. Dietary pattern analysis: a new direction in nutritional epidemiology. Current Opinion in Lipidology 2002; 13: 3-9.

6 Huijbregts P, Feskens E, Rasanen L, Fidanza F, Nissinen A, Menotti A, et al. Dietary pattern and 20 year mortality in elderly men in Finland Italy, and the Netherlands: longitudinal cohort study. British Medical Journal 1997; 315: 13-7.

7 Kant AK, Schatzkin A, Ziegler RG. Dietary diversity and subsequent cause-specific mortality in the NHANES I Epidemiologic Follow-up Study. Journal of the American College of Nutrition 1995; 14: 233-8.

8 Kant AK, Schatzkin A, Graubard BI, Schairer C. A prospective study of diet quality and mortality in women. Journal of the American Medical Association 2000; 283: 2109-15.
9 Trichopoulou A, Costacou T, Bamia C, Trichopoulos D. Adherence to a Mediterranean diet and survival in a Greek population. New England Journal of Medicine 2003; 348: 2599-608.

10 US Department of Agriculture, US Department of Health and Human Services. Nutrition and Your Health: Dietary Guidelines for Americans. Washington, DC: US Government Printing Office, 1995.

11 US Department of Agriculture, US Department of Health and Human Services. Dietary Guidelines for Americans 2005, 6th ed. Washington, DC: US Government Printing Office, 2000.

12 US USDA's Food Guide, Background and Development. Department of Agriculture. Washington, DC: USDA, Human Information Service, 1993.

13 Kennedy ET, Ohls J, Carlson S, Fleming K. The Healthy Eating Index: design and applications. Journal of the American Dietetic Association 1995; 95: 1103-8.

14 McCullough ML, Feskanich D, Stampfer MJ, Rosner BA, Hu FB, Hunter DJ, et al. Adherence to the Dietary Guidelines for Americans and risk of major chronic disease in women. American Journal of Clinical Nutrition 2000; 72: 1214-22.

15 McCullough ML, Feskanich D, Rimm EB, Giovannuci EL, Ascherio A, Variyam JN, et al. Adherence to the Dietary Guidelines for Americans and risk of major chronic disease in men. American Journal of Clinical Nutrition 2000; 72 : $1223-31$.

16 Mensink RPM, Katan MB. Effect of dietary trans fatty acids on high-density and low-density lipoprotein cholesterol levels in healthy subjects. New England Journal of Medicine 1990; 323: 439-45.

17 Katan MB, Grundy SM, willett WC. Should a low-fat, highcarbohydrate diet be recommended for everyone? Beyond low-fat diets. New England Journal of Medicine 1997; 337: 563-7.

$18 \mathrm{Hu} F B$, Stampfer MJ, Manson JE, Rimm EB, Colditz GA, Rosner BA, et al. Dietary fat intake and the risk of coronary heart disease in women. New England Journal of Medicine 1997; 337: 1491-9.

19 de Lorgeril M, Salen P, Martin JL, Manjaud I, Boucher P, Mamelle N. Mediterranean dietary pattern in a randomized trial. Prolonged survival and possible reduced cancer rate. Archives of Internal Medicine 1998; 158: 1181-7.

20 Kolonel LN. Fat, meat and prostate cancer. Epidemiologic Reviews 2001; 23: 72-81.

21 Klatsky AL, Armstrong MA, Friedman GD. Risk of cardiovascular mortality in alcohol drinkers, ex-drinkers, and nondrinkers. American Journal of Cardiology 1990; 66: $1237-42$.

22 McCullough ML, Feskanich D, Stampfer MJ, Giovannucci EL, Rimm EB, Hu FB, et al. Diet quality and major chronic disease risk in men and women: moving toward improved dietary guidance. American Journal of Clinical Nutrition 2002; 76: 1261-71.

23 Willett WC, Sampson L, Stampfer MJ, Rosner B, Bain C, Witschi $\mathrm{J}$, et al. Reproducibility and validity of a semiquantitative food frequency questionnaire. American Journal of Epidemiology 1985; 122: 51-65.

24 Feskanich D, Rimm EB, Giovannucci EL, Colditz GA, Stampfer MJ, Litin LB, et al. Reproducibility and validity of food intake measurements from a semiquantitative food frequency questionnaire. Journal of the American Dietetic Association 1993; 93: 790-6.

25 Rimm EB, Giovannucci EL, Stampfer MJ, Colditz GA, Litin LB, Willett WC. Reproducibility and validity of a expanded selfadministered semiquantitative food frequency questionnaire among male health professionals. American Journal of Epidemiology 1992; 135: 1114-26.

26 Giovannucci E, Colditz G, Stampfer MJ, Rimm EB, Litin L, Sampson L, et al. The assessment of alcohol consumption by 
a simple self-administered questionnaire. American Journal of Epidemiology 1991; 133: 810-7.

27 Salvini S, Hunter DJ, Sampson L, Stampfer MJ, Colditz GA, Rosner B, et al. Food-based validation of a dietary questionnaire: the effects of week-to-week variation in food consumption. International Journal of Epidemiology 1989; 18: 858-67.

28 Michaud DS, Giovannucci EL, Ascherio A, Rimm EB, Forman MR, Sampson L, et al. Associations of plasma carotenoid concentrations and dietary intake of specific carotenoids in samples of two prospective cohort studies using a new carotenoid database. Cancer Epidemiology, Biomarkers $\mathcal{E}$ Prevention 1998; 7: 283-90.

29 Rose GA, Blackburn H. Cardiovascular survey methods. In: WHO Monograph Series No. 58. Geneva: World Health Organization, 1982.

30 Walker AE, Robins M, Weinfeld FD. The national survey of stroke. Clinical findings. Stroke 1981; 12: I13-I44.

31 Stampfer MJ, Willett WC, Speizer FE, Dysert DC, Lipnick R, Rosner B, et al. Test of the National Death Index. American Journal of Epidemiology 1984; 119: 837-9.

$32 \mathrm{Hu}$ FB, Stampfer MJ, Rimm E, Ascherio A, Rosner BA, Spiegelman D, et al. Dietary fat and coronary heart disease: a comparison of approaches for adjusting for total energy intake and modeling repeated dietary measurements. American Journal of Epidemiology 1999; 149: 531-40.
33 Cupples LA, D'Agostino RB, Anderson K, Kannel WB. Comparison of baseline and repeated measure covariate techniques in the Framingham Heart Study. Statistics in Medicine 1988; 7: 205-22.

34 D'Agostino RB, Lee MLT, Belanger AJ, Cupples LA, Anderson $\mathrm{K}$, Kannel WB. Relation of pooled logistic regression to time dependent Cox regression analysis: The Framingham Heart Study. Statistics in Medicine 1990; 9: 1501-15.

35 Sacks F. Dietary fats and coronary heart disease. Overview. Journal of Cardiovascular Risk 1994; 1: 3-8.

36 Kushi LH. Health implications of the Mediterranean diets in light of contemporary knowledge. 2. Meat, wine, fats and oils. American Journal of Clinical Nutrition 1995; 61: $1416 \mathrm{~S}-27 \mathrm{~S}$.

37 Giovannucci E. Tomatoes, tomato-based products, lycopene, and cancer: review of the epidemiologic literature. Journal of the National Cancer Institute 1999; 91: 317-31.

38 Baron JA, Beach M, Mandel JS, van Stolk RU, Haile RW, Sandler RS, et al. Calcium supplements for the prevention of colorectal adenomas. New England Journal of Medicine 1999; 340: 101-7.

39 Platz EA, Giovannucci E. Vitamin D and calcium in colorectal and prostate cancers. In: Heber D, Blackburn GL, Go VLW, eds. Nutritional Oncology. San Diego, CA: Academic Press, 1999; 223-52. 